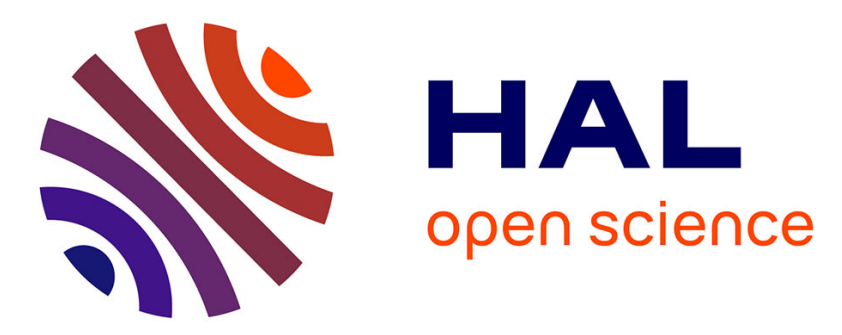

\title{
Dynamic beam deflection using photorefractive gratings in Bi12SiO20 crystals
}

\author{
Gilles Pauliat, Jean-Pierre Herriau, Anne Delboulbé, Gérald Roosen, \\ Jean-Pierre Huignard
}

\section{- To cite this version:}

Gilles Pauliat, Jean-Pierre Herriau, Anne Delboulbé, Gérald Roosen, Jean-Pierre Huignard. Dynamic beam deflection using photorefractive gratings in Bi12SiO20 crystals. Journal of the Optical Society of America B, 1986, 3 (2), pp.306-314. hal-00867496

\section{HAL Id: hal-00867496 \\ https://hal-iogs.archives-ouvertes.fr/hal-00867496}

Submitted on 30 Sep 2013

HAL is a multi-disciplinary open access archive for the deposit and dissemination of scientific research documents, whether they are published or not. The documents may come from teaching and research institutions in France or abroad, or from public or private research centers.
L'archive ouverte pluridisciplinaire HAL, est destinée au dépôt et à la diffusion de documents scientifiques de niveau recherche, publiés ou non, émanant des établissements d'enseignement et de recherche français ou étrangers, des laboratoires publics ou privés. 


\title{
Dynamic beam deflection using photorefractive gratings in $\mathrm{Bi}_{12} \mathrm{SiO}_{20}$ crystals
}

\author{
G. Pauliat \\ Institut d'Optique, Bât. 503, Laboratoire Associé au Centre National de la Recherche Scientifique, Centre \\ Universitaire-BP 43, 91406 Orsay Cédex, France \\ J. P. Herriau and A. Delboulbé \\ Thomson-CSF, Laboratoire Central de Recherches, Domaine de Corbeville-BP 10, 91401 Orsay Cédex, France
}

\author{
G. Roosen
}

Institut d'Optique, Bât. 503, Laboratoire Associé au Centre National de la Recherche Scientifique, Centre Universitaire-BP 43, 91406 Orsay Cédex, France

\section{J. P. Huignard}

Thomson-CSF, Laboratoire Central de Recherches, Domaine de Corbeville-BP 10, 91401 Orsay Cédex, France

Received August 10, 1985; accepted October 11, 1985

\begin{abstract}
New configurations for spatial beam steering of a near-infrared laser beam are presented. They are based on the interaction of the beam from a semiconductor laser with a dynamic grating photoinduced in a photorefractive crystal such as $\mathrm{Bi}_{12} \mathrm{SiO}_{20}$ (BSO). The demonstrated large values of the deflection angles $\left(12^{\circ}\right)$ result from a change in both the grating period and the orientation achieved by optical means. The Bragg condition over this large scan angle is maintained by either recording the grating with a variable wavelength (dye laser) or taking advantage of the spatial nonlinearities of the photorefractive effect. The respective advantages of these two techniques are discussed. Possible application to interconnection between optical fibers for broadband communication is considered.
\end{abstract}

\section{INTRODUCTION}

The recording and reading of optical gratings in photorefractive materials ${ }^{1}$ have been widely investigated in the past few years. These materials have already shown good potential for applications in optical processing, phase conjugation with gain, image amplification, ${ }^{2-7}$ etc. All these experiments are based on degenerate two-wave and four-wave mixing configurations, i.e., configurations for which all the interacting beams have nearly the same wavelength. The purpose of this paper is to show another type of application in which the photorefractive crystal is considered an active optical element allowing spatial switching of a near-infrared beam that is either emitted by a semiconductor laser or emerges from a multimode or single-mode fiber cable. As presented in the preliminary work in Refs. 8 and 9, these laser beam-steering techniques would potentially permit large deflection angles and millisecond random-access time. We present and analyze in this paper two optical addressing methods. that permit large deflection angles $\left(\delta \theta \simeq 10-15^{\circ}\right)$ maintaining the Bragg condition. Through the association of a large number of switching elements, these techniques could be an attractive solution to the important problem of the optical interconnection between fiber matrices. ${ }^{10,11}$

\section{PRESENTATION OF THE PHOTOREFRACTIVE MATERIALS FOR DYNAMIC SPATIAL SWITCHING}

\section{A. General Introduction}

The principle of deflection described in this paper is based on some specific properties of the photorefractive materials, in particular, bismuth silicon oxide crystals, $\mathrm{Bi}_{12} \mathrm{SiO}_{20}$ (BSO). In what follows, we summarize the main properties of these recording media in view of the envisaged application.

The materials usually employed for holography, such as high-resolution silver plates and dichromated gelatin, are not adapted to the dynamic switching of optical beams. Photothermoplastics are erasable materials, but their number of recording-erasure cycles is limited to about $10^{2}-10^{3} \cdot{ }^{12-13}$ Therefore photorefractive crystals are now the most attractive candidates for this purpose. In particular, $\mathrm{BSO}$ [or bismuth germanate (BGO) and bismuth titanate (BTO)] is a promising material on account of its high sensitivity in the blue-green spectral range, high optical quality, and availability of large crystals. The complete erasure of a photoinduced grating is obtained by uniform illumination of 
the crystal (generally with the same wavelength as used for recording). Since these nonlinear materials are sensitive to the incident energy (100-500 $\mu \mathrm{J} \mathrm{cm}^{-2}$ for an elementary grating at $\lambda=514.5 \mathrm{~nm}$ ), the grating recording time constants are typically $10 \mathrm{msec}$ for an incident power density of 10-50 $\mathrm{mW} \mathrm{cm}^{-2}$ (Ref. 14) and may become as short as 10 nsec when recording with a pulse laser providing $10-50 \mathrm{~kW}$ $\mathrm{cm}^{-2}{ }^{15,16}$ This crystal exhibits a very low sensitivity in the near infrared (IR) $\left(750 \mathrm{~nm}<\lambda_{R}<1600 \mathrm{~nm}\right)$, and, typically, the readout time constant reaches $2 \mathrm{~h}$ at the wavelength $\lambda_{R}$ $\simeq 840 \mathrm{~nm}$ for an incident intensity $I_{R} \simeq 60 \mu \mathrm{W} \mathrm{cm}{ }^{-2}$. (The dark storage time is about 20-30 h. ${ }^{14}$ ) Since there is a change of wavelength between recording $(\lambda \simeq 500 \mathrm{~nm})$ and readout $\left(\lambda_{R} \simeq 840 \mathrm{~nm}\right)$, the incidence of the readout beam must be positioned at the Bragg angle. ${ }^{17}$ The objective of this paper is to demonstrate the deflection of an IR beam over large scan angle $\left(\delta \theta \simeq 10^{\circ}-15^{\circ}\right)$ by changing the spacing $\Lambda$ of a photoinduced grating in the BSO by optical means. This requires new methods for maintaining the Bragg-diffraction conditions for a fixed incidence $\theta_{R}$ of the IR beam.

\section{B. The Photorefractive Effect in Electro-Optic Crystals}

The light-induced refractive-index change in these materials is due to the existence of charges located in low-lying traps formed by impurities or to defect sites in the crystal. ${ }^{1}$

In absence of light, the charges are frozen in place by the low dark conductivity of the material. However, under illumination the trapped charges can migrate between trapping sites. The hopping model ${ }^{18}$ assumes that carrier transport occurs by electron hopping from a filled donor site to an enpty trap located nearby. The problem is then discussed in terms of the hopping probability, the hopping rate being proportional to the local optical intensity. The band transport model ${ }^{19}$ considers that carriers, say, electrons, are optically excited from filled donor sites to the conduction band. These carriers then migrate by diffusion and drift before recombining into an empty trap. With a nonuniform sinu-

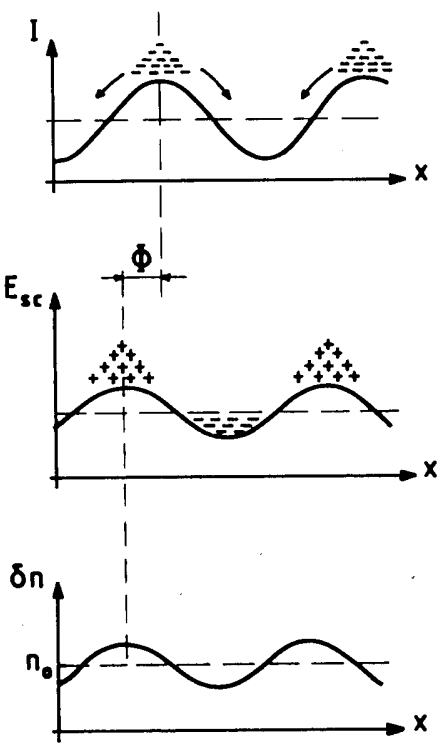

(a)

(b)

Fig. 1. Basic principle for grating recording mechanism in photorefractive crystals. (a) Illumination-carrier photoexcitation and charge transport (drift or diffusion); (b) phase-shifted photoinduced space-charge field; (c) related refractive-index modulation. soidal illumination, this process creates a periodic photoinduced space-charge electric field that modulates the crystal refractive index by the linear electro-optic effect (Fig. 1). The photoinduced phase volume grating persists after illumination for seconds, or for months, depending on the dark conductivity of the material. It can be read quasi-nondestructively with a light beam of wavelength outside the crystal sensitivity range, in the near IR, for example. The phase grating is erasable under uniform illumination; photocarriers are thus reexcited and uniformly redistributed, giving rise to a relaxation of the index modulation. The time constant for grating writing or erasure varies as the inverse of the incident beam intensities. ${ }^{20}$

\section{Grating Efficiency versus the Recording Parameters}

The illumination resulting from the interference of two beams of intensities $I_{1}$ and $I_{2}$, respectively, is

$$
I=I_{0}[1+m \cos (\mathbf{K} x)],
$$

where $m$ is the modulation index, $m=2\left[\left(I_{1}+I_{2}\right)^{1 / 2}\right] / I_{0}$, with $I_{0}=I_{1}+I_{2} ; \mathbf{K}$ is the grating wave vector, $\mathbf{K}=2 \pi / \Lambda$; and $\Lambda$ is the grating spacing, $\Lambda=\lambda / 2 \sin \theta$.

The electric-field variation $\delta E$ photoinduced by the modulated part of the illumination produces a change in the refractive index given by

$$
\delta n=\frac{n_{0}{ }^{3} r_{41} \delta E}{2},
$$

where $r_{41}$ is the electro-optic coefficient related to the crystal orientation shown in Fig. 2 (grating wave vector parallel to the 110 direction); $r_{41} \simeq 3.6 .10^{-12} \mathrm{~m} \mathrm{~V}^{-1} .^{21}$

The photoinduced space-charge field amplitude in the steady state given by Kukhtarev et al. ${ }^{19}$ is related to the material and experimental recording parameters by the following expression:

$$
E_{\mathrm{sc}}=m\left[\frac{E_{0}{ }^{2}+E_{D}{ }^{2}}{\left(1+\frac{E_{D}}{E_{Q}}\right)^{2}+\frac{E_{0}{ }^{2}}{E_{Q}{ }^{2}}}\right]^{1 / 2},
$$

where $E_{D}$ is the diffusion field, $E_{D}=k T \mathbf{K} / e$, and $E_{Q}$ is the maximum space-charge field amplitude that can be photoinduced in the crystal, $E_{Q}=e N_{A}+/ \epsilon_{0} \epsilon_{r} \mathbf{K} . \quad \epsilon_{0}$ is the free-space permittivity, $\epsilon_{r}$ is the static relative dielectric constant, and $N_{A}{ }^{+}$is the density number of active trap sites in the crystal, $N_{A}{ }^{+} \simeq 5 \times 10^{15} \mathrm{~cm}^{-3}$.

The buildup time constant of the grating derived from Refs. 20 and 7 is given by

$$
\begin{aligned}
\tau_{\omega}= & \tau_{i} \times\left(\frac{\mu \tau k T}{e}\right)^{2} \times \frac{E_{Q}}{E_{D}} \\
& \times \frac{\left[\mathbf{K}^{2}+\left(\frac{e}{\mu \tau k T}\right)^{2}\right]^{2}+\left(\frac{\mathbf{K} e E_{0}}{k T}\right)^{2}}{\left(1+\frac{E_{Q}}{E_{D}}\right)\left[\mathbf{K}^{2}+\left(\frac{e}{\mu \tau k T}\right)^{2}\right]+\left(\frac{\mathbf{K} e E_{0}}{k T}\right)^{2}},
\end{aligned}
$$

where $\tau_{i}$ is the dielectric relaxation time under incident illumination $I_{0}, \mu$ is the charge mobility, $\tau$ is the photoelectron lifetime, and $E_{0}$ is the applied electric field. The scattering efficiency of the photoinduced grating is given by ${ }^{17}$ 

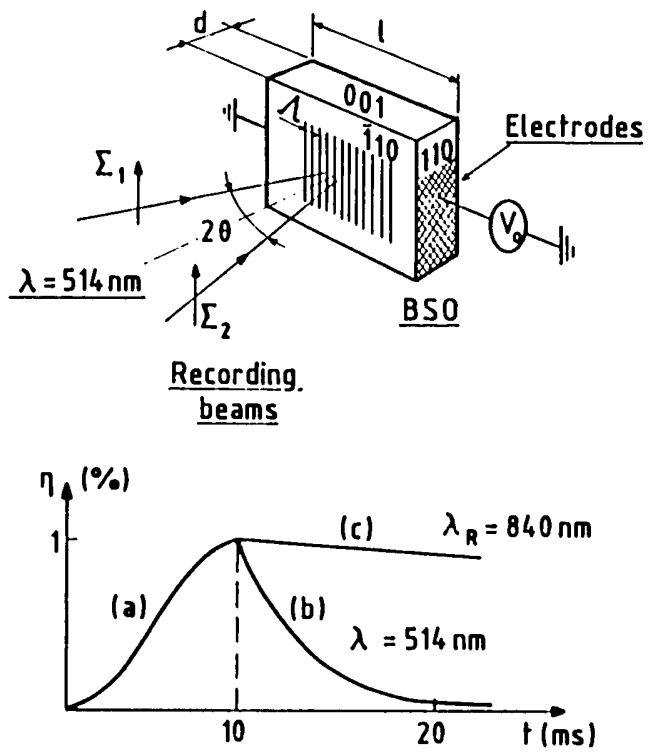

Fig. 2. Electro-optic configuration for grating recording in BSO crystals. Typical recording, erasure cycles, spacing $\Lambda=4 \mu \mathrm{m}$, applied field $E_{0}=6 \mathrm{kV} \mathrm{cm}^{-1}$. (a) Grating recording, $I_{0}=20 \mathrm{~mW} \mathrm{~cm}^{-2}$, $\lambda=514 \mathrm{~nm}$; (b) grating erasure, $\lambda=514 \mathrm{~nm}$; (c) grating readout in the near IR. $\lambda_{R}=840 \mathrm{~nm}$; erasure time constant, $\tau=2 h$ for $I_{R}=60$

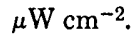

$$
\eta=\exp (-\alpha d) \sin ^{2}\left(\frac{\pi d}{2 \lambda_{R} \cos \theta_{R}} n_{0}{ }^{3} r_{41} E_{\mathrm{sc}}\right),
$$

where $d$ is the crystal length, $\theta_{R}$ is the Bragg angle inside the crystal, $\lambda_{R}$ is the wavelength of the readout beam, and $\alpha$ is the absorption coefficient.

These relations predict an efficiency of $\eta \simeq 100 \%$, but the efficiencies measured with BSO are quite far from these theoretical limits. A typical grating recording-erasure cycle is given in Fig. 2, for which the steady-state diffraction efficiency is $\eta_{s} \simeq 1 \%$ for $E_{0} \simeq 6 \mathrm{kV} \mathrm{cm} \mathrm{cm}^{-1}$, a crystal thickness $d=5 \mathrm{~mm}$, and a grating spacing $\Lambda \simeq 2 \mu \mathrm{m}$. Improved results may be expected from appropriate doping of the crystal. For example, larger values of the efficiency may be reached if the density of the traps is increased slightly.

\section{LIGHT BEAM STEERING THROUGH DYNAMIC GRATING RECORDING IN BSO}

In the application considered here the IR beam keeps a fixed incidence $\theta_{R}$ upon the crystal, and its deflection is achieved through the variation of the photoinduced grating spacing $\Lambda$. In order to maintain optimum efficiency over a large scan angle, the following two parameters have to be precisely controlled:

(1) The grating spacing $\Lambda$ that fixes the direction of deflection of the IR beam.

(2) The slant $\varphi$ of the grating in the volume of the BSO to place the grating planes under the correct incidence with respect to the readout beam.

To satisfy these requirements, two original methods are presented in the following subsections.

\section{A. Variable-Wavelength Recording for Bragg Tracking}

1. Description of the Method

In order to simplify notation, we do not express the dependence of the following parameters on the writing wavelength: $\varphi, \Lambda, \gamma, i_{1}, i_{2}, n, \theta_{1}, \theta_{2}, \theta_{R}^{B}, \theta_{R}{ }^{\prime}$, and $i_{R}^{\prime}$. The maximum deflection efficiency is obtained for the fundamental grating period when the incident recording beams have nearly equal intensity $(m \simeq 1) .^{19}$ This grating is induced in the photorefractive material by interference of two coherent plane waves, at wavelength $\lambda$, emitted by a dye laser. Changing the recording wavelength $\lambda^{22}$ produces a modification of the grating period $\Lambda$, which thus gives rise to a modification of the diffraction direction of the beam at the wavelength $\lambda_{0}$, according to the following relation (Fig. 3):

$$
\sin \left(\theta_{R}+\varphi\right)-\sin \left(\theta_{R}^{\prime}-\varphi\right)= \pm \frac{\lambda_{R}}{n_{0} \Lambda}
$$

where $\theta_{R}$ and $\theta_{R}^{\prime}$ are, respectively, the incident and the emerging angles of the reading beam, $\varphi$ is the tilt of the fringe pattern, and $n_{0}$ is the refractive index of the crystal at wavelength $\lambda_{R}$.

For efficient diffraction, the Bragg condition has to be satisfied:

$$
\theta_{R}=\theta_{R}{ }^{B}, \quad \theta_{R}^{\prime}=\theta_{R}^{B^{\prime}}
$$

with

$$
\sin \left(\theta_{R}{ }^{B^{\prime}}-\varphi\right)=-\sin \left(\theta_{R}{ }^{B}+\varphi\right)=\frac{\lambda_{R}}{2 n_{0} \Lambda} .
$$

As a consequence, when the grating spacing is varied, the fringe-pattern orientation must be simultaneously tilted.

Therefore, in order to achieve efficient switching toward a given direction, the dynamic grating has to be recorded with the correct set of parameters $(\Lambda, \varphi)$. Since the control parameter is the wavelength of the recording beams, the simultaneous change in spacing and orientation of the induced grating, demonstrated by Sincerbox and Roosen, ${ }^{9}$ is reached in the following way: On the path of one of the recording beams is placed a dispersive element $H_{1}$ (Fig. 4a). A shift in the control wavelength $\lambda$ produces a corresponding change in the incident angle of this recording beam. By proper choice of the parameters of $H_{1}$, this passive and easy method permits a phase hologram appropriate for Bragg diffraction of the beam at $\Lambda_{R}$ to be recorded in the photorefractive

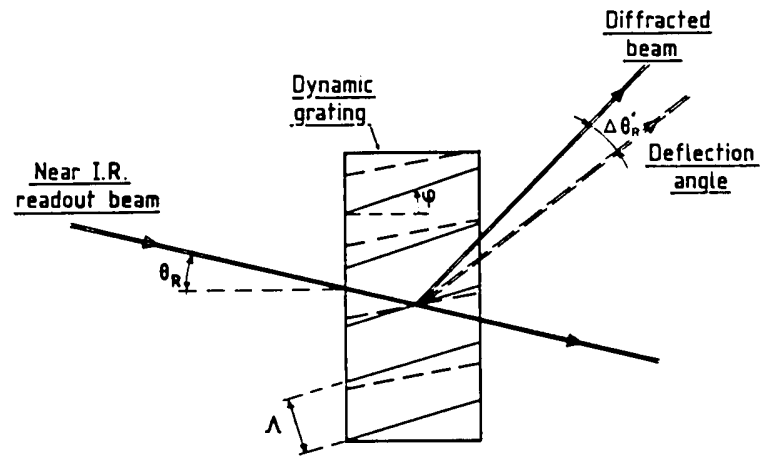

Fig. 3. Principle of light-beam deflection by a dynamic volume grating photoinduced in a photorefractive crystal. The deflection is achieved by the variation of the grating period $\Lambda$ and orientation $\varphi$. 

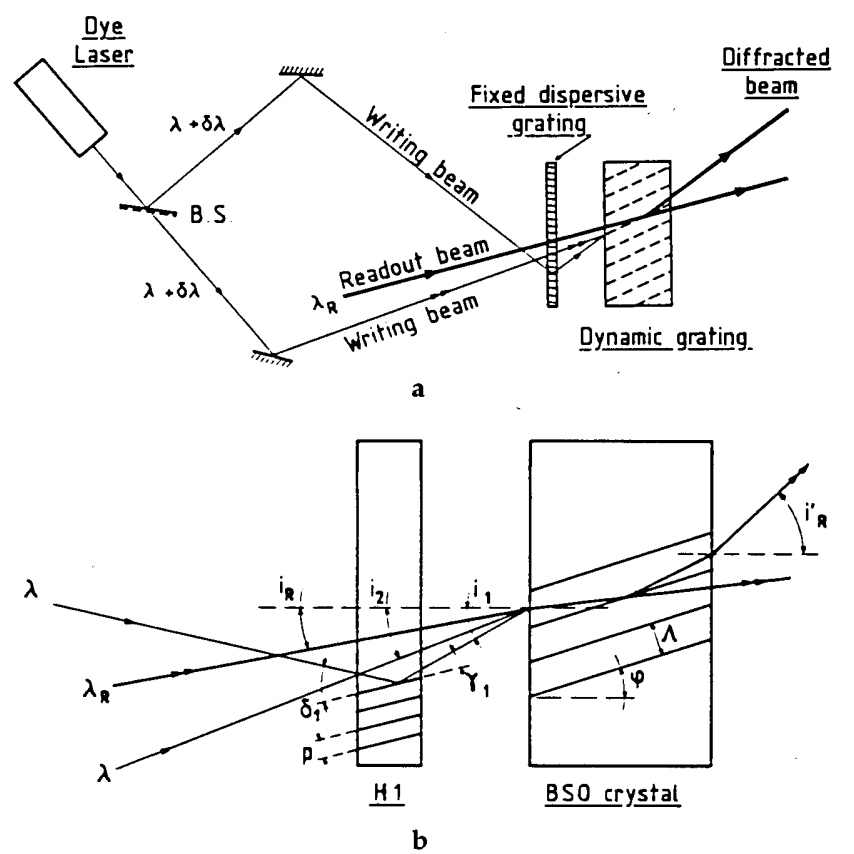

Fig. 4. Schematic of an elementary switching cell based on the recording with a variable wavelength (laser dye). Association of a fixed dispersive grating (dichromated gelatin) with a photorefractive BSO. a, A writing beam is incident at the Bragg angle, and the dispersive grating provides a change in both the period and the orientation of the dynamic grating when the wavelength varies. B.S., Beam splitter. b, Notation relative to the switching cell.

crystal for any wavelength $\lambda$. As a consequence, this reading beam experiences very large deflections and efficient diffraction. Numerous configurations have been defined, any of them offering specific advantages. For example, the easiest recording method is at zero applied field, but in this case the diffraction efficiency remains low with a maximum for a grating period $\Lambda \simeq 1 \mu \mathrm{m}$. On the other hand, grating spacing $\Lambda \geq 4 \mu \mathrm{m}$ provides an enhancement of the efficiency $(10 \times)$, but at the expense of a large applied field $(>6 \mathrm{kV}$ $\left.\mathrm{cm}^{-1}\right) .^{23}$

\section{Parameters of an Elementary Switching Cell}

In order to realize a compact switching cell, the dispersive element $H_{1}$ is a high-efficiency grating permanently recorded in dichromated gelatin and placed in close contact with the photorefractive crystal. For easy implementation, the fringe orientations of both the fixed and the dynamic gratings are chosen to be nearly perpendicular to the crystal surface. For the control beam incident at the Bragg angle, $H_{1}$ presents a large diffraction efficiency over the entire range of writing wavelengths $(\lambda=530-575 \mathrm{~nm})$. On the other hand, it is completely transparent for the other two beams. Since we have chosen to work in the diffusion mode, the average period of the dynamic grating is taken to be equal to $\Lambda \simeq 1 \mu \mathrm{m}$, with the readout beam at normal incidence upon the crystal. The optimum period $p$ of the fixed grating that satisfies the Bragg condition over the largest wavelength range is determined by solving the grating equation for both the fixed and the dynamic holograms. The set of equations is shown below (Fig. $4 \mathrm{~b}$ ).
For the recording beams we have

$$
\begin{aligned}
\sin \gamma_{1} & = \pm \frac{1}{p} \lambda_{1}-\sin \delta_{1}, \\
i_{1} & =\gamma_{1}+A_{1} .
\end{aligned}
$$

The internal angles in the medium are

$$
\begin{aligned}
& \theta_{1}=\sin ^{-1}\left(\frac{1}{n_{1}} \sin i_{1}\right), \\
& \theta_{2}=\sin ^{-1}\left(\frac{1}{n_{1}} \sin i_{2}\right),
\end{aligned}
$$

where $n_{i}$ is the refractive of the material for wavelength $\lambda$.

Thus the spacing $\Lambda$ and the orientation $\varphi$ of the photoinduced grating are

$$
\begin{aligned}
& \Lambda=\frac{\lambda}{2 n \sin \left(\frac{\theta_{1}-\theta_{2}}{2}\right),} \\
& \varphi=1 / 2\left(\theta_{1}+\theta_{2}\right) .
\end{aligned}
$$

The equations that govern Bragg diffraction of the reading beam at $\lambda_{R}$ are

$$
\begin{aligned}
\theta_{R}{ }^{B} & =\varphi-\sin ^{-1}\left(\frac{\lambda_{R}}{2 n_{0} \Lambda}\right), \\
\theta^{\prime} & =\varphi+\sin ^{-1}\left(\frac{\lambda_{R}}{2 n_{0} \Lambda}\right),
\end{aligned}
$$

The external angles are thus

$$
\begin{aligned}
i_{R} & =\sin ^{-1}\left(n_{0} \sin \theta_{R}\right), \\
i_{R}^{\prime} & =\sin ^{-1}\left(n_{0} \sin \theta^{\prime}\right) .
\end{aligned}
$$

The Bragg detuning is then

$$
\delta \theta=\theta_{R}^{B}-\theta_{R}
$$

At this point, it is possible to devise the switching cell such that the reading beam is either incident at the exact Bragg

Table 1. Bragg Detuning versus Recording Wavelength ${ }^{a}$

\begin{tabular}{ccc}
\hline $\begin{array}{c}\text { Writing } \\
\text { Wavelength } \\
\lambda(\mathrm{nm})\end{array}$ & \multicolumn{2}{c}{ Bragg Detuning $\delta \theta$ (min) } \\
\cline { 2 - 3 } 532 & Fig. 5(a) & Fig. 5(b) \\
\hline 535 & $-2.0^{\prime}$ & $-1.0^{\prime}$ \\
540 & $-1.5^{\prime}$ & $-0.5^{\prime}$ \\
545 & $0.0^{\prime}$ & $0.0^{\prime}$ \\
550 & $0.0^{\prime}$ & $1.0^{\prime}$ \\
555 & $0.0^{\prime}$ & $1.0^{\prime}$ \\
560 & $0.0^{\prime}$ & $1.0^{\prime}$ \\
565 & $-1.0^{\prime}$ & $0.0^{\prime}$ \\
570 & $-1.5^{\prime}$ & $-0.5^{\prime}$ \\
\hline
\end{tabular}

${ }^{a}$ Hologram thickness, $d=2.74 \mathrm{~mm}$; period of the fixed grating, $p=0.36 \mu \mathrm{m}$. ${ }^{b}$ Without detuning of the mean wavelength $(550 \mathrm{~nm})$.

c With detuning of $1 \mathrm{~min}$ at the mean wavelength. 


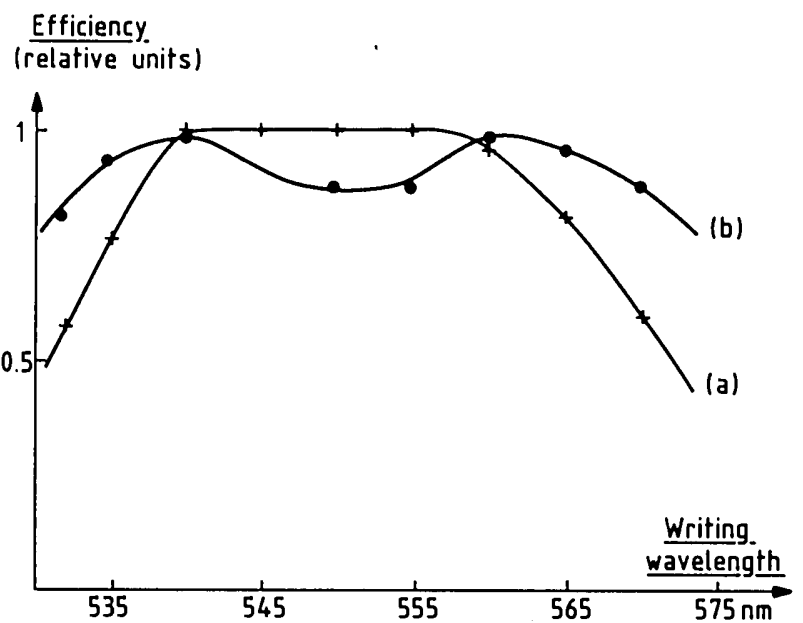

Fig. 5. Experimental variation of the diffraction efficiency versus the writing wavelength. Readout wavelength $\lambda_{R}=840 \mathrm{~nm} . \Lambda=1$ $\mu \mathrm{m}$ at $\lambda_{i}=550 \mathrm{~nm}$. Period of the dispersive grating $p=0.36 \mu \mathrm{m}$. (a) Zero Bragg detuning at $\lambda=550 \mathrm{~nm}$; (b) $0.3-\mathrm{mrad}$ Bragg detuning at $\lambda=550 \mathrm{~nm}$.

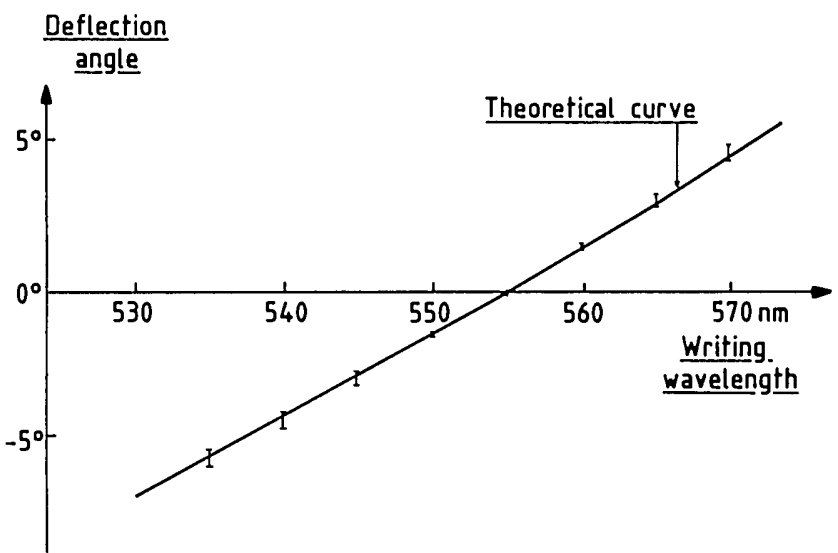

Fig. 6. Deflection angle of the near-IR beam versus the recording wavelength of the dye laser. Comparison between the theoretical and the experimental points.

angle on the grating photoinduced at the mean writing wavelength or presents a small detuning. This mismatch in turn decreases the Bragg detuning on the gratings recorded at wavelengths on the edge of the allowed wavelength range and consequently gives an increase in the width of the efficiency curve. However, this increase occurs at the expense of the efficiency in the middle of the curve, which exhibits a slight dip. Such a comparison is given in the Table 1 (hologram thickness, $d=2.74 \mathrm{~mm}$; period of the fixed grating, $p=$ $0.36 \mu \mathrm{m})$.

\section{Results}

The relative diffraction efficiency as a function of the recording wavelength is presented in Fig. 5. As mentioned previously, a slight Bragg detuning on the grating recorded at the mean wavelength permits one either to enlarge the allowed wavelength shift or to maintain a higher diffraction efficiency for a given wavelength range [Fig. 5(b)]. As far as deflection is concerned, Fig. 6 demonstrates the good agreement between theoretical predictions and experimental results and confirms how the fringe-tilting technique permits large deflections to be produced by achieving an efficient tracking of the Bragg condition. The slope of the curve gives a deflection sensitivity of about $0.28^{\circ} \mathrm{nm}^{-1}$. For the experimental adjustment of Fig. 5(b), i.e., a Bragg detuning of $0.3 \mathrm{mrad}$ on the grating recorded at the mean wavelength, this corresponds to a measured deflection of $\delta \theta^{\prime}{ }_{R} \simeq 11.4^{\circ}$ over the available wavelength range $(532 \leq \lambda \leq 572 \mathrm{~nm})$. For such a deflection range, the diffraction efficiency remains larger than $75 \%$ of its maximum value. For the present experiment, the scan of the laser wavelengths was achieved by mechanical rotation of a birefringent filter. However, for fast wavelength changes and random access, electro-optically driven filters can be used. ${ }^{24}$

In order to address the maximum of separate directions, the important parameter to consider is the number of resolvable spots defined as

$$
N_{P} \leq \frac{\Delta \theta_{R}^{\prime}}{\delta \theta_{R}^{\prime}}
$$

where $\Delta \theta^{\prime}{ }_{R}$ and $\delta \theta_{R}^{\prime}$ are, respectively, the reading-beam deflection angle and the beam divergence measured inside the crystal.

In this experiment the divergence angle of the IR beam at $\lambda_{R} \simeq 840 \mathrm{~nm}$ was slightly smaller than $1 \mathrm{mrad}$. Considering the Rayleigh criterion, this gives

$$
N_{p} \leq 200 \text {, }
$$

which is quite an acceptable number for these applications. Figure 7 shows the intensity distribution of the IR beam after switching. Figure 7(a) shows four different diffracted beams corresponding to four writing wavelengths. A variation $\delta \lambda=1 \mathrm{~nm}$ of the wavelength of the recording beam $\lambda$ produces two very well separated diffracted beams. In this

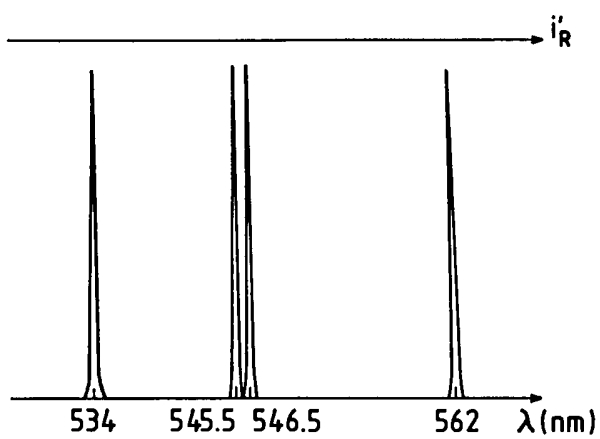

(a)

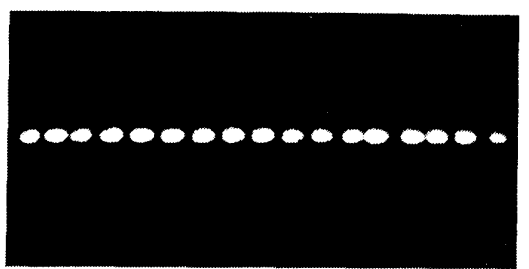

Fig. 7. (a) Intensity distribution in the deflected beam focused onto a linear detector array. Four different writing wavelengths (redrawn from oscilloscope trace). (b) Photograph of a part of the deflected spots. 


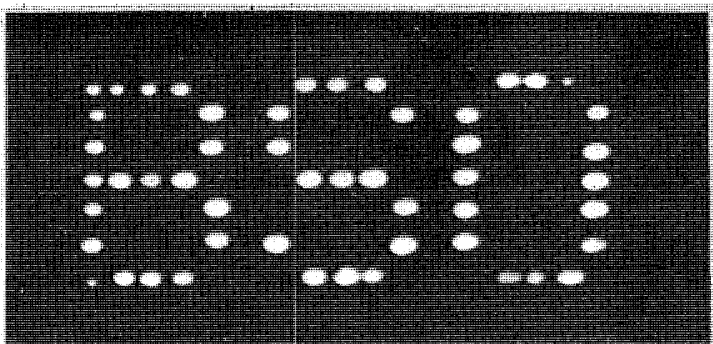

Fig. 8. Two-dimensional beam deflection achieved by combination of the variable recording-wavelength technique (horizontal axis) and the displacement of one recording beam (vertical axis).

experiment, conducted without an external applied electric field, the diffraction efficiency was low $\left(\eta \simeq 10^{-3}\right.$ for a crystal thickness of $d=2.74 \mathrm{~mm}$ ). This value can be improved by 1 order of magnitude when an applied electric field is used in combination with larger grating periods $(\Lambda \geq$ $4 \mu \mathrm{m}$ ). The switching time was about $\tau_{\omega} \simeq 100 \mathrm{msec}$ for a power density of $10 \mathrm{~mW} \mathrm{~cm}^{-2}$ at the mean recording wavelength $\lambda \simeq 550 \mathrm{~nm}$. This switching time can be reduced to about $10 \mathrm{msec}$ by using a more appropriate dye, emitting the same power around $\lambda \simeq 500 \mathrm{~nm}$, which is the wavelength of greatest sensitivity for BSO.

When one of the recording beams is moved out of the plane of Fig. 4, it results in a tilt of the photoinduced grating, thus producing a deflection of the near-IR beam out of the figure plane. Therefore, in combination with the variable recording-wavelength technique, this permits two-dimensional beam steering, as demonstrated in Fig. 8.

\section{B. Collinear Bragg Diffraction}

1. Description of the Method

The preservation of the Bragg condition whatever the spatial frequency and the grating orientation in the BSO may be also obtained with a method based on the nonlinear mixing of two independent gratings. ${ }^{25}$ These two gratings (grating vectors $\mathbf{K}_{1}$ and $\mathbf{K}_{2}$ ) are photoinduced by a proper choice of two collinear beams at wavelengths $\lambda_{1}$ and $\lambda_{2}$, and the readout in the near IR (wavelength $\lambda_{R}$ ) is exactly collinear to the direction of the writing beams. The basic principle of the method is shown in Fig. 9. We consider in this figure the recording of two independent gratings resulting from the interference in the same direction of two plane waves at wavelength $\lambda_{1}$ and $\lambda_{2}$. The resulting intensity distributions are given, respectively, by

$$
\begin{array}{cc}
I_{1}=I_{0_{1}}\left[1+\cos \left(\mathbf{K}_{1} x\right)\right], & \mathbf{K}_{1}=\frac{4 \pi \sin \theta}{\lambda_{1}}, \\
I_{2}=I_{0_{2}}\left[1+\cos \left(\mathbf{K}_{2} x\right)\right], & \mathbf{K}_{2}=\frac{4 \pi \sin \theta}{\lambda_{2}} .
\end{array}
$$

The expressions of the photoinduced space-charge field and of the related index change under sinusoidal light exposure given in Section 2 are essentially valid for a low value of the incident fringe modulation $(m \ll 1)$. These solutions are essentially linear. For the case of a grating recording with an externally applied electric field $E_{0}\left(E_{0} \gg E_{D}\right)$ and grating spacings such as $E_{0}<E_{Q}(\Lambda \simeq 6 \mu \mathrm{m}$ typically), the photoinduced space-charge field is simply given by

$$
E_{\mathrm{sc}}=m E_{0} \cos (\mathbf{K} x) \quad m<1 .
$$

For modulation amplitudes $m$ approaching unity, as used in this experiment, the crystal response is nonlinear, and a more complete analytic expression of the photoinduced space-charge field at saturation derived from Ref. 26 is given by

$$
E_{\mathrm{sc}}=E_{0} \frac{\left(1-m_{1}^{2}\right)^{1 / 2}}{1+m_{1} \cos (\mathbf{K} x)},
$$

where $m_{1}=m \sigma_{0} /\left(\sigma_{I}+\sigma_{0}\right) . \quad \sigma_{0}$ is the crystal dark conductivity and $\sigma_{I}$ is the conductivity of the crystal under incident illumination.

This relation does not account for the saturation of the space-charge field amplitude versus the externally applied field $E_{0}$ resulting from the complete filling of the traps in BSO (or BGO and BTO) crystals. Nevertheless, we will use that closed-form expression of the field in order to point out the nonlinearities of the crystal response. In the experiment considered in Fig. 9, the photoinduced space-charge field resulting from the crystal illumination by the two incoherent light patterns $I_{1}$ and $I_{2}$ having a fringe modulation amplitude of nearly unity may be described by the following relation deduced from Eq. (25):

$$
E_{\mathrm{sc}}(x)=E_{0} \frac{\left(1-m_{1}^{2}\right)^{1 / 2}}{1+1 / 2 m_{1}\left(\cos \mathbf{K}_{1} x+\cos \mathbf{K}_{2} x\right)} .
$$

This relation may be expanded into Fourier series and can take the form:

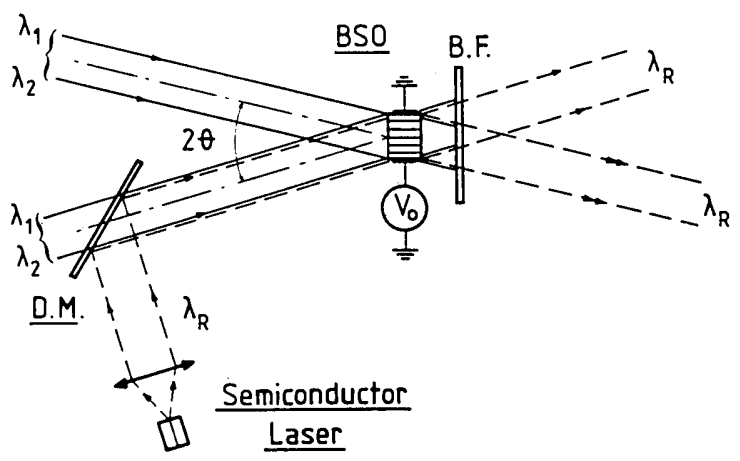

Fig. 9. Principle of collinear Bragg diffraction in photorefractive BSO crystals. Crystal size, $10 \mathrm{~mm} \times 10 \mathrm{~mm}$; thickness, $10 \mathrm{~mm} ; V_{0}=$ $6 \mathrm{kV}$; beam angle, $2 \theta=10^{\circ}$. Writing wavelengths, $\lambda_{1}=632.8 \mathrm{~nm}$ and $\lambda_{2}=514.5 \mathrm{~nm}$; readout wavelength, $\lambda_{R} \simeq 840 \mathrm{~nm}$; B.F., blocking filter for $\lambda_{1}$ and $\lambda_{2}$; D.M., dichroic mirror.

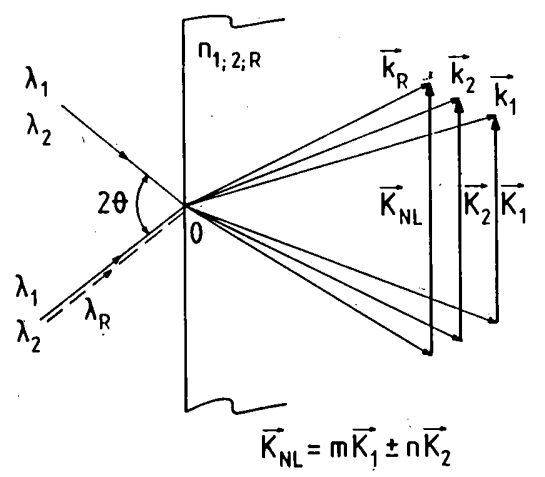

Fig. 10. Wave-vector diagram illustrating the collinear Bragg diffraction based on the spatial nonlinearities of the photorefractive effect. Grating wave vector $\mathbf{K}_{\mathrm{NL}}$ results from the nonlinear mixing of the grating wave vectors $\mathbf{K}_{1}, \mathbf{K}_{2}$. 

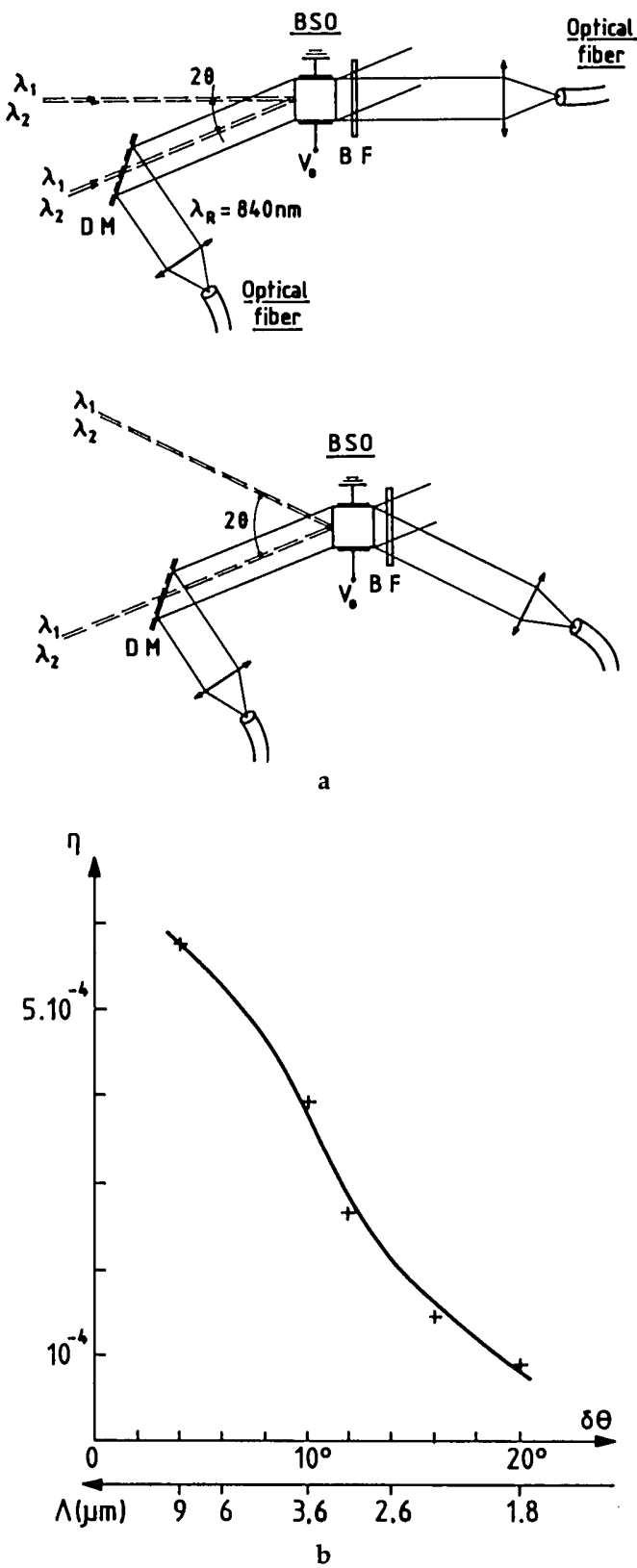

Fig. 11. a, Application of the collinear Bragg diffraction in BSO to the steering of a light beam issuing from an optical fiber. Wavelength $\lambda_{R}=840 \mathrm{~nm}$. Dashed lines, direction of the two writing beams $\left(\lambda_{1}=632.8 \mathrm{~nm}, \lambda_{2}=514 \mathrm{~nm}\right)$. b, Evolution of the deflected beam intensity in collinear Bragg diffraction versus the scan angle. $E_{0}=8 \mathrm{kV} \mathrm{cm}^{-1}$, BSO crystal thickness $d=7 \mathrm{~mm}$.

$$
\begin{aligned}
E_{\mathrm{sc}}(x)= & \frac{E_{0}}{2} \sum_{n=0}^{\infty} \sum_{m=0}^{\infty} \alpha_{m, n} \\
& \times\left\{\cos \left[\left(m \mathbf{K}_{1}+n \mathbf{K}_{2}\right)\right] x+\cos \left[\left(m \mathbf{K}_{1}-n \mathbf{K}_{2}\right) x\right]\right\}
\end{aligned}
$$

Therefore exposing the crystal with the two collinear grating wave vectors $\mathbf{K}_{\mathbf{1}}$ and $\mathbf{K}_{2}$ generates through nonlinear mixing the new wave vectors $\mathbf{K}_{\mathrm{NL}}=m \mathbf{K}_{1} \pm n \mathbf{K}_{2}$, which satisfy the Bragg condition for a readout wavelength $\lambda_{R}$ such as

$$
\frac{1}{\lambda_{R}}=\frac{m}{\lambda_{1}} \pm \frac{n}{\lambda_{2}}
$$

The wave-vector diagram corresponding to such a nonlinear interaction is given in Fig. 10. This geometrical construction illustrates that the Brágg condition is fulfilled, thus permitting an exact collinearity of the incident recording $\left(\lambda_{1}, \lambda_{2}\right)$ and diffracted $\left(\lambda_{R}\right)$ beams. By a proper choice of $m$ and $n$ in the last relation, a near-IR readout wavelength can be obtained while keeping the incident recording wavelengths $\lambda_{1}$ and $\lambda_{2}$ in the spectral sensitivity range of the BSO crystal.

\section{Experimental Results}

The experimental demonstration of the nonlinear mixing of the $\mathbf{K}$ vectors is achieved with the two incident recording wavelengths: $\lambda_{1}=632.8 \mathrm{~nm}$ (helium-neon laser) and $\lambda_{2}=$ $514.5 \mathrm{~nm}$ (argon laser). Choosing the values of the parameters $m$ and $n$ equal to $m=2$ (for $\lambda_{1}$ ) and $n=1$ (for $\lambda_{2}$ ), respectively, ensures a collinear Bragg diffraction at a wavelength $\lambda_{R}=822 \mathrm{~nm}$. This wavelength is close to the emission wavelength of the semiconductor laser that we have used for the experiment $\left(\lambda_{R}=840 \mathrm{~nm}\right)$. The measured diffraction efficiency is $\eta=5 \times 10^{-4}$ for an applied electric field $E_{0}=6 \mathrm{kV} \mathrm{cm}^{-1}$ and a grating spacing $\Lambda \simeq 10 \mu \mathrm{m}$. The grating formation time is short with the green line of the argon laser (typically $\tau_{\omega} \simeq 10 \mathrm{msec}$ for $I_{01}=10 \mathrm{~mW} \mathrm{~cm}^{-2}$ ), but it reaches a few seconds for the recording with the red line of the helium-neon laser. Certainly this last time constant is not convenient for beam-steering applications, but the first objective was to demonstrate the validity of the method using available laser sources. Other wavelengths such as $\lambda_{1}=560 \mathrm{~nm}$ and $\lambda_{2}=420 \mathrm{~nm}(m=1 ; n=2)$ would be better adapted for short response times. The application of this recording technique to light-beam steering is shown in Fig. 11a. When the incidence of the visible recording beams is changing by $10^{\circ}$ in the two $X$ and $Y$ directions, the IR beam is diffracted under the Bragg condition and is exactly collinear to the incident recording beams. The evolution of the diffraction efficiency related to this scan angle is shown in Fig. 11b.

\section{DISCUSSION AND CONCLUSION}

Concerning the diffraction efficiency, which is one of the important parameters for fiber-to-fiber connection, one can conclude that the variable-wavelength recording method is more efficient. Indeed, the collinear Bragg method is based on the nonlinear mixing of higher-order nonlinearities, and it consequently results in lower efficiency. However, both techniques would require materials having greater photoinduced index variation and a short response time. Photorefractive ferroelectric crystals such as $\mathrm{BaTiO}_{3}$ and $\mathrm{KNbO}_{3}$ certainly have the required index modulation because of their high electro-optic coefficient, but the time response is long. 3,7 On the other hand, materials such as BSO and BGO have the required speed, but the index variation should be increased. This would require optimization (and identification) of the trap density and/or appropriate doping during crystal growth.

These dynamic holographic techniques can be the active element of interconnection devices ensuring communication exchanges between two-dimensional arrays of optical fibers. ${ }^{10}$ The experiments presented that permit the dynamic spatial switching of optical beams in the near-IR region 


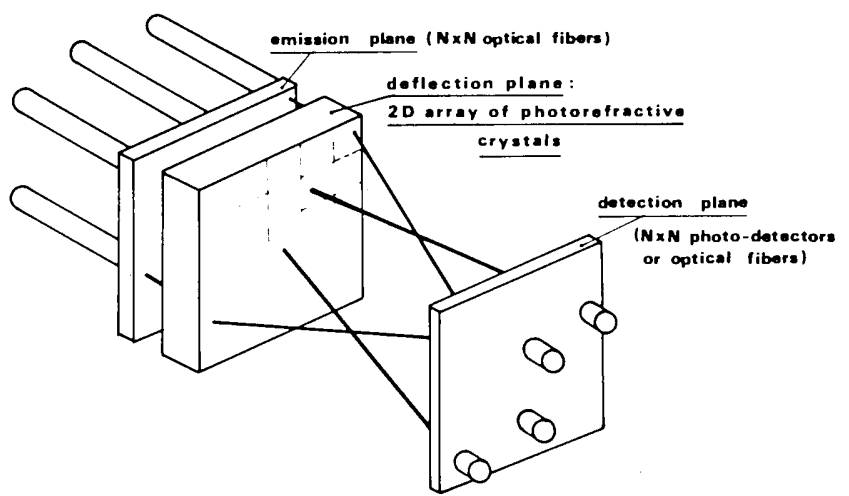

Fig. 12. Principle of a spatial switching device for fiber-to-fiber interconnection based on the two-dimensional association of elementary deflection cells.

appear extremely attractive in this field. Because of the memory effect of the photorefractive effect, many channels can be connected in parallel with no limitation in bandwidth as soon as the grating is recorded. However, the design of a complete switching device, whose schematic is given in Fig. 12 , requires the extension of the principles described here to a two-dimensional array of photorefractive switching cells as well as two-dimensional deflection of the IR beam for each cell. With view to accomplishing such a project, further studies on both material optimization and system architecture must be pursued.

\section{ACKNOWLEDGMENTS}

The authors are grateful to P. Gravey and J. Y. Moisan (Centre National d'Etudes des Telecommunications-Lannion) and to S. Fioreze and A. Rey (Thomson-CSF, Telephone) for very helpful discussions. They älso express their thanks to the Direction des Affaires Industrielles et Internationales (Ministère des P.T.T) for partial support of this research.

\section{REFERENCES}

1. A. M. Glass, Opt. Eng. 17, 470 (1978); D. L. Staebler, in Holographic Recording Materials, H. M. Smith, ed. (Springer-Verlag, Berlin, 1977), pp. 101-132.

2. J. P. Huignard, H. Rajbenbach, Ph. Refregier, and L. Solymar, Opt. Eng. 24, 586 (1985).

3. S. I. Stepanov and M. P. Petrov, Opt. Acta 31, 1335 (1984).

4. J. Feinberg and R. W. Hellwarth, Opt. Lett. 5, 519 (1980).

5. M. Cronin-Golomb, B. Fisher, J. O. White, and A. Yariv, IEEE J. Quantum Electron. QE-20, 12 (1980).

6. P. Gunter, Phys. Rep. 93, 199 (1982).

7. G. Valley and M. Klein, Opt. Eng. 22, 704 (1983).

8. P. D. Henshaw, Appl. Opt. 21, 2323 (1982).

9. G. T. Sincerbox and G. Roosen, Appl. Opt. 22, 690 (1983).

10. J. W. Goodman, F. J. Leonberger, and S. Y. Kung, Proc. IEEE 27, 850 (1984).

11. P. Gravey, in Proceedings of the 7th E.C.O.C. 81 (University Press, Copenhagen, 1981).

12. U. Killat and D. R. Terrel, Opt. Acta 24, 441 (1977).

13. J. Y. Moisan, P. Gravey, R. Lever, and L. Bonnel, in Proceedings

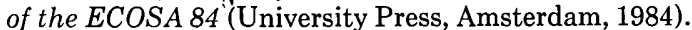

14. J. P. Huignard and F. Micheron, Appl. Phys. Lett. 29, 591 (1976).

15. J. P. Hermann, J. P. Herriau, and J. P. Huignard, Appl. Opt. 20, $2173(1980)$

16. G. Lesaux, G. Roosen, and A. Brun, Opt. Commun. (to be published).

17. H. Kogelnik, Bell Syst. Tech. J. 48, 2909 (1969).

18. J. Feinberg, D. Heiman, A. R. Tanguay, and R. W. Hellwarth, J. Appl. Phys. 51, 1297 (1980).

19. N. Kukhtarev, V. Markov, S. Odulov, M. Soskin, and V. Vinetskii, Ferroelectrics 22, 949 (1979).

20. N. Kukhtarev, Sov. Tech. Phys. Lett. 2, 438 (1976).

21. P. Pellat Finet, Opt. Commun. 50, 275 (1984).

22. G. Roosen and M. T. Plantegenest, Opt. Commun. 14, 199 (1983).

23. J. P. Huignard, J. P. Herriau, G. Rivet, and P. Gunter, Opt. Lett. 5, $102(1980)$.

24. J. M. Telle and C. L. Tang, Appl. Phys. Lett. 26, 358 (1983).

25. J. P. Huignard and B. Ledu, Opt. Lett. 7, 310 (1982).

26. M. G. Moharam, T. K. Gaylord, R. Magnusson, and L. Young, J. Appl. Phys. 50, 5642 (1979). 


\section{G. Pauliat}

G. Pauliat graduated from the Université Paris XI, Orsay, France, in 1984. He is preparing a $\mathrm{PhD}$. dissertation on the photorefractive materials and their applications with G. Roosen at the Institut d'Optique Théorique et Appliquée.

\section{J. P. Herriau}

J. P. Herriau was born in Paris on October 16, 1945. He graduated from Conservatoire National des Arts et Métiers, Paris, in 1976. Since 1970 he has been working at Thomson-CSF Research Laboratories in Orsay. His research interests have included ellipsometry, electro-optic crystals and ceramics, interferometry, metrology, and holography. His current interests include photorefractive crystals and interconnections. Dr. Herriau is a member of the International Society for Optical Engineering and the Sqciété Française d'Optique.

\section{A. Delboulbé}

Delboulbé was born on August 7, 1961. She received the technical degree in optics from the Lycee Fresnel, Paris, in 1981. She joined the optical group of Thomson-CSF Research Laboratories in Orsay in September 1981. She participates in experimental studies on photorefractive crystals applied to optical signal processing and ințerconnections.

\section{G. Roosen}

G. Roosen graduated from the Université Paris XI, Orsay, France, in 1969. He received the Doctorat ès-Sciences (physics) from this university in 1978. He is Chargé de Recherches, Centre National de la Recherche Scientifique, and works at the Institut d'Optique Théorique et Appliquée. From 1971 to 1979 he worked on the mechanical effects produced by light radiation pressure, including optical levitation. He spent a year as a visiting scientist at the IBM Research Laboratory in San Jose, California. His research interests include both physics and applications of optical nonlinearities and subpicosecond pulses. Dr. Roosen is currently responsible for nonlinear optics in photorefractive and semiconductor materials at the Institut d'Optique. He is a member of the Société Française d'Optique, the Optical Society of America, and the International Society for Optical Engineering.

\section{J. P. Huignard}

J. P. Huignard was born on August 28, 1944. He is a graduate in physics from the Université Paris XI, Orsay, and of the Ecole Supérieure d'Optique (Institut d'Optique, Orsay). His research interests have included optical information storage, electro-optics crystals and devices, and holographic optical elements and recording materials. His current interests include optical phase conjugation, electro-optics, photorefractive crystals, and related applications to optical signal processing. Dr. Huignard is a member of the Optical Society of America, the Society of Photo-Optical and Instrumentation Engineers, and the Société Française d'Optique. 